\title{
Thiamine Deficiency and Benfotiamine Therapy in Brain Diseases
}

\author{
Dragan M Pavlović* \\ Department of Psychology, University of Belgrade, Serbia
}

*Corresponding author: Dragan M Pavlović, Professor of Neurology and Neuropsychology. Faculty for special education and rehabilitation, University of Belgrade, St. Visokog Stevana 2, 11000 Belgrade, Serbia.

To Cite This Article: Dragan M Pavlović. Thiamine Deficiency and Benfotiamine Therapy in Brain Diseases. Am J Biomed Sci \& Res. 2019 - 3(1). AJBSR.MS.ID.000621. DOI: 10.34297/AJBSR.2019.03.000621

Received: April 30, 2019 | Published: May 08, 2019

\begin{abstract}
Thiamine is hydrosoluble molecule from B vitamins family vitamin that is crucial for the metabolism of glucose and is essential for the normal growth and development of the organism, as well as for the normal function of body systems such as digestive, cardiovascular and nervous system, both central (CNS) and peripheral (PNS), and also stimulates the brain and improves the psycho-emotional state. Hypovitaminosis B1 is more common than it is thought, primarily due to the discrepancy in high intake of pure calories and the low intake of vitamins. The most frequent deficiencies of thiamine are Wernicke's encephalopathy, Korsakoff's psychosis, beriberi, Alzheimer's disease, but many more cases are those of marginal deficit of thiamine.

Benfotiamine is a synthetic vitamin B1 that is liposoluble than manifold increases his penetration in brain and peripheral nerves compared to thiamine. Benfotiamine is thiamine precursor that can be converted into thiamine in tissues and then metabolites into thiamine monophosphate and thiamine diphosphate. Polyphenols in coffee and tea can inactivate thiamine.

The effects of benfotiamine are mild inhibition of cholinesterase, reduction in production of amyloid plaques and hyperphosphorylated tau. Benfotiamine inhibits the three main pathways leading to hyperglycemic damage. Long-term administration of benfotiamine corrects cognitive status. Usual doses of benfotiamine are $150 \mathrm{mg}$ or $300 \mathrm{mg}$ once or twice daily. In some cases, Korsakoff psychosis requires long-term administration of benfotiamine in maintenance dose with excellent results.
\end{abstract}

Keywords: Thiamine; Benfotiamine; Wernicke’s Encephalopathy; Korsakoff's Psychosis; Beriberi; Alzheimer’s Disease

\section{Introduction}

Vitamin B1 (aneurin, thiamine) is a hydrosoluble vitamin that is crucial for the metabolism of glucose and is essential for the normal growth and development of the organism, as well as for the normal function of body systems such as digestive, cardiovascular and nervous system, both central (CNS) and peripheral (PNS), and also stimulates the brain and improves the psycho-emotional state [1]. Chemically, vitamin B1 consists of a pyrimidine ring (2,5-dimethyl6-aminopyridine) and a thiazoline ring (4-methyl-5-hydroxyethyl thiazole) which are linked by a methylene bridge [2].

Benfotiamine is a synthetic vitamin B1 that is liposoluble than manifold increases his penetration in brain and peripheral nerves compared to thiamine. In comparative studies it has been shown that lipophilic thiamine derivatives are significantly better resorbed than hydrophilic thiamine [3] In comparison with thiamine, benfotiamine has better resorption and bioavailability. Benfotiamine is thiamine precursor that can be converted into thiamine in tissues and then metabolites into thiamine monophosphate and thiamine diphosphate [4]. Transformation of benfotiamine into thiamine produces hydropuric acid in significant amounts that is secreted by urine. The recommended daily amount of dietary allowance (RDA) is $0.5 \mathrm{mg}$ per $1000 \mathrm{kcal}$. Significant amounts of vitamin B1 are lost during thermal treatment of food. Polyphenols in coffee and tea can inactivate thiamine.

Vitamin B1 cannot be accumulated in the body, so signs of deficiency are rapidly displayed [1]. Thiamine stores in the body are only about $30 \mathrm{mg}$, with a half-life of 10-18 days [5]. Hypovitaminosis B1 is seen in chronic alcoholism, persistent vomiting (like in some pregnant women) or after bariatric surgical procedures, but in mild form also in the general population [6].

\section{Function}

Vitamin B1 is essential for the normal function of central nervous system (CNS), peripheral nervous system (PNS) and heart and is part of the body's important enzymes. Vitamin B1 allows 
normal use of glucose as well as carbohydrates and proteins and provides the supply of energy to the body [6]. Thiamine-dependent enzymes use thiamin diphosphate (ThDP) as coenzyme [7]. These enzymes are also called ThDP-dependent enzymes. Other thiamine derivatives are thiamine triphosphate and thiamine adenylate, and they participate in homeostasis, which is called non-enzymatic activity of vitamin B1 [7].

The thiamine excess is stored in small amounts in the liver and erythrocytes in the form of thiamine pyrophosphate (TPP) which is derived from thiamine pyrophosphorylation using adenosine triphosphate (ATP). In case of lack of thiamine, the TPP from the stock is hydrolyzed to thiamine monophosphate, which is further hydrolyzed to thiamine and released into circulation [2]. There is a large number of thiamine transporter in biological membranes. Thiamine is degraded by thiaminases produces by microorganisms in the intestines.

Cholinergic neurons have a greater requirement for thiamine than other cells and are sensitive to its deficit. This is due to the high need for acetyl-CoA both for the production of energy and for the synthesis of various molecules [8].

\section{Thiamine deficiency}

Common data: Hypovitaminosis B1 is more common than it is thought, primarily due to the discrepancy in high intake of pure calories and the low intake of vitamins, with the situation worsening by the fact that this deficiency is a major imitator [2]. Historically, it was first described by beriberi in Japan, several centuries ago, and in the 19th century by Wernicke's encephalopathy and Korsakoff's syndrome. A modern diet with "fast" foods favor the occurrence of the deficiency of vitamin B1. The aggravating factor is the lack of pathognomonic signs and reliable laboratory diagnostics. A study conducted by Williams in the United States in 1943 showed a significant spread of vitamin B1 deficiency in the general population [9]. Typically, thiamine deficiency is present in Alzheimer's disease (AD).

Affected systems: The most commonly affected systems in the lack of thiamine are heart, blood vessels, CNS, PNS, eyes and gastrointestinal system [10]. Periventricular gray matter neurons are particularly sensitive to the lack of thiamine [11]. Damage to the nuclei of the vestibular nerve and abducens nerve may precede encephalopathy, which should be kept in mind in order to apply thiamine supplements in a timely manner and prevent the onset of severe forms of hypovitaminosis such as beriberi, Wernicke's encephalopathy and Korsakoff's syndrome.

The deficiency of thiamine produces pseudohypoxia at the cellular level, and later to dysautonomia in various tissues [2]. In the case of B1 hypovitaminosis, lactates, pyruvates and other substances increase in the serum. The lack of thiamine disturbs the function of nitric oxide (NO) which is an important transmitter, especially in the vascular system.

Beriberi: The lack of vitamin B1 causes a disease of beriberi with weight loss, emotional disorders, changes in perception, weakness of the extremities, irregular heartbeat and tissue edema. Heart failure may lead to fatal outcome. No sign of beriberi is pathognomonic, and the clinical picture is very variable [2]. There is a classification on dry beriberi (neurological) with prominent polyneuropathy and wet beriberi (cardiac) with dominate cardiomyopathy with peripheral edema, although they are often associated [10]. There are also gastrointestinal complaints.

Beriberi was first described in Japan by people who used white rice. Thus, the food contained a high level of calories with the lack of other essential nutrients called high calorie malnutrition [12]. Today, high-calorie malnutrition is not a rarity in the West in obese people where thiamine deficiency is present in as much as $15-29 \%$ of people with mild and non-specific symptoms [2]. Beriberi can be divided into alcohol-induced and non-alcoholic form. Increased risk is in people taking diuretics such as furosemide that enhance the secretion of vitamin B1 [5].

Wernicke-Korsakoff syndrome: Wernicke's encephalopathy and Korsakoff's dementia/ psychosis are often interconnected phenomena caused by the lack of vitamin B1 [14]? If occurring together, the condition is called the Wernicke-Korsakoff syndrome. Both disorders require urgent treatment, but the diagnosis is often postponed or not made at all. Autopsy findings in terms of these diseases are much more common than prevalence in the general population and in alcoholics [14]. Korsakoff's psychosis is a severe acute dementia with the inability to memorize new information, anterograde amnesia and confabulations [10]. Korsakov's psychosis is a typical example of diencephalic amnesia. In the Wernicke-Korsakoff syndrome, there is a hyperemic damage to the brain, hypothalamus, thalamus, and mammary bodies, gliosis, capillary dilatation, and perivascular bleeding [10]. Wernicke's encephalopathy is expressed as global confusion, disturbance of the oculomotors (nystagmus, ophthalmoplegia) and ataxia, which is a classic triad that, however, is seen in only $16-20 \%$ of cases, so the diagnosis is often wrong [14]. It is particularly difficult to diagnose the condition in patients with confusion cause by alcohol abuse or other causes, the use of benzodiazepines, sepsis, hypoxia, hepatic encephalopathy, delirium tremens, and head injuries. Untreated Wernicke's encephalopathy has lethal outcome in about $20 \%$ of cases [15]. Most commonly, Wernicke's encephalopathy and/or Korsakoff's dementia occur in chronic alcoholics, and then in decreasing frequency in carcinoma, gastrointestinal surgery, Hyperemesis gravidarum, fasting, gastrointestinal diseases, AIDS, disorders of resorption, dialysis and kidney disease, parenteral nutrition, vomiting, psychiatric diseases with eating disorders, schizophrenia, infections, intoxication, thyroid disorders, iatrogenic states, in cases of poor nutrition, hypoxic encephalopathy, diarrhea, magnesium deficiency, some congenital conditions and other states [14]. There is also an inborn defect of the SLC19A3 gene that leads to deficiency of thiamine transporeter-2 [16]. In about $81 \%$ of the published cases of Wernicke's encephalopathy, the reason was not alcohol, and in 28\%, Korsakov's syndrome developed [17]. On the magnetic resonance of the brain, symmetrical T2 hyperintensities in the dorsal medial thalamus, mammillary bodies, periaqueductal gray mass and in the tectum were observed in Wernicke's encephalopathy [16]. However, $13 \%$ of patients had a normal MRI [17]. 
The hereditary disorders of thiamine metabolism: Multiple mutations lead to disorders that respond favorably to thiamine administration. The pyruvic dehydrogenase complex (PDHC) consists of three enzymes: decarboxylase, dihydrolipoyl transacetylase which produces acetyl Coenzyme A (CoA) and dihydrolipoyl dehydrogenase which produces NADH. Mutations in any component of this complex can lead to disturbances of citric acid cycle and insufficient energy generation in mitochondria, which appears on the periphery as lactoacidosis [2]. There is a shortage of acetylcholine, ataxia, proximal weakness, etc. Thiamine phosphokinase (TPK) converts thiamine into the active form - thiamine pyrophosphate (TPP). Mutations of this enzyme are described which lead to ataxia, psychomotor retardation and dystonia. There is lactoacidosis and a decrease in the concentration of TPP. Leigh's disease is called subacute necrotizing encephalomyelopathy and is partially responsible for the administration of thiamine. Disease mutations on thiamine transporter genes have also been described. Thyamine-dependent megaloblastic anemia is a rare disease with diabetes mellitus (DM) and sensorineural deafness [18]. Therapeutic use of vitamin B1 alleviates symptoms.

Neurodegenerative diseases: The chronic lack of vitamin B1 significantly contributes to the development of neurodegenerative diseases [1]. Thiamin is associated with AD, Parkinson's disease (PD), and Huntington's disease (HD). Thiamine administration not only acts neuroprotective but also has a beneficial effect even in advanced neurodegenerative diseases.

In a study, three cases of patients with newly diagnosed $\mathrm{PD}$, were given $100 \mathrm{mg}$ of thiamine parenterally twice a week, that led to significant regression of motor symptoms [19]. Blood thiamine levels were initially not reduced, indicating a disorder of intracellular mechanisms.

The lack of vitamin B1 is a risk factor for $\mathrm{AD}$ and is reduced in this disorder [20]. Early studies have shown that levels of thiamine diphosphate (TDP), and TDP-dependent enzymes are significantly lowered in blood and brain samples of people with AD [21]. A possible link between the lack of thiamine and $\mathrm{AD}$ is a disorder of glucose metabolism that can be found for decades before the first clinical manifestations of cognitive decline [20]. Thyamine deficiency is thought to promote the creation of A beta plaques, neurofibrillary tangles (NFK), and neuronal loss in the medial temporal lobe [22]. Pathophysiological changes and cognitive decline due to lack of vitamin B1 have been confirmed on animal models [23].

Marginal deficit of thiamine in adults: Mild forms of thiamine deficiency are frequent because supplies are scarce (liver, muscle) and can occur after 2-3 weeks after thiamine-deficient nutrition [10]. Alcohol, tea and coffee lower thiamine levels as well as higher amounts of carbohydrates. Also at the risk are people with an increased need for thiamine, such as pregnant women, during lactation, people exposed to great physical efforts, suffering from cancer, liver, infections and hyperthyroidism, patients undergoing surgical procedures, as well as disorders of absorption (alcohol, gastrointestinal diseases, diarrhea, vomiting). The deficiency of thiamine in these mild case can be expressed as anorexia, malaise, paresthesia in the extremities distal, irritability and depression, and later, after 2-3 months, insomnia, cough, fatigue, emotional lability, panic attacks, joint and muscle pain, increased need for sugar, gastro-esophageal reflux, abdominal pain, daytime headache or migraine, nose mucous membrane edema, dyspnea, tiredness, paresthesia, cold extremities, heart palpitation, chest pain, constipation and diarrhea, intolerance of ambient temperature and repetitive nausea and vomiting $[2,10]$. Anorexia is a possible protective mechanism to prevent carbohydrate intake and further loss of thiamine. Neurologically, polyneuropathy is most commonly encountered with paresthesia, especially in legs, reduced muscle stretch reflexes and progressive muscular weakness with increased susceptibility to infection [10]. The lack of thiamine cannot always be proven, but often elevated triglycerides, fibrinogen and hypersensitivity CRP as markers of inflammation can be found [24].

Deficiency of thiamine in children and adolescents: The lack of thiamine in children should be suspected in the full spectrum of non-specific symptoms such as: oxidative stress, emotional, behavioral and attention disorders, learning disorders, facial redness, paleness around the mouth, blood pressure disorders, muscle stretch reflex disorders, dermography etc. [2]. The acute manifestation of thiamine deficiency in infants is rarely rare but difficult and with high mortality. The child is upset, has constipation, it is crying, with generalized edema, dyspnea, cardiac disorders and oliguria. Cyanosis, paralysis of speech with aphonia and pseudomeningitis with neck stiffness, vomiting, convulsions and sweating can sometimes occur.

\section{Diagnosis}

The testing of vitamin B1 is based on the measurement of concentrations of serum thiamine pyrophosphate or transketolase activity (TKA; thiamine-dependent enzyme) in erythrocytes [6] and thiamin pyrophosphate effect (TPPE) [10]. The best method is probably high-performance liquid chromatography (HPLC) for the determination of thiamine diphosphate, monophosphate and thiamine [21].

Well documented cases of Wernicke's encephalopathy with normal or even elevated levels of thiamine have been described [16]. This is probably the consequence of discrepancies in serum and tissue levels, as is the case with vitamin B12 and magnesium $[2,10]$. Normal serum thiamine levels are 70-180 nmol/L, and the deficit is less than $70 \mathrm{nmol} / \mathrm{L}$. It is probably more reliable to determine TKA in erythrocytes that better reflects the tissue content, and the normal range is from $42.1-86.1 \mathrm{mF} / \mathrm{L} / \mathrm{min}$, or for TPPE normal $0-14 \%$. For the TPPE, the border deficit is $15-24 \%$, and the deficit is $25+\%$ [10].

\section{Therapy}

Areas of use: Thiamin is used in patients with anxiety disorders, chronic lymph nodes, sleep disorders, anorexia, nausea, digestive disorders, chest and abdominal pain, depression, aggression, headaches, etc. Some studies have shown a beneficial effect of vitamin B1 in people with AD and PD [7]. The importance of thiamine administration in the Wernicke-Korsakoff syndrome, 
beriberi and other deficiencies is unequivocal. Instantaneous replacement of thiamine is necessary to prevent irreversible changes [14]. Because of the urgency, vitamin B1 is usually used empirically, especially since blood B1 level in the blood is not a reliable measure of deficiency.

There are nogenerally accepted guidelines for the administration of thiamine. There are schemes for giving thiamine for prophylactic purposes in persons at risk with $100 \mathrm{mg}$ intramuscularly three times daily for 3-5 days or $250 \mathrm{mg}$ intramuscularly for 3-5 days, as well as many other schemes [14]. In diagnosed Wernicke and/ or Korsakoff's syndrome, thiamine intravenous is administered at doses of 100-500 mg for at least 5 days, and then less doses, e.g. 250 mg intramuscularly until the occurrence of improvement. Usually long-term oral administration is required in order to achieve better effects.

The excess of thiamine is excreted via the kidney [10]. Individual adverse events have been reported in intravenous administration at doses of 5-100 mg, and very rarely an allergic reaction can occur at extremely high oral doses of 5-10 g [25]. Administration of thiamine at doses of $500 \mathrm{mg}$ per day does not lead to toxic effects [26].

Benfotiamine: The effects of benfotiamine are mild inhibition of cholinesterase, reduction in production of amyloid plaques and hyperphosphorylated tau (the basis for the formation of NFT) [27]. It also accelerates the recovery of peripheral nerves after various types of damage. In an animal model, the administration of benfotiamine improved spatial memory in a dosagedependent manner and reduced both A-beta plaques and levels of hyperphosphorylated tau, whereas other forms of thiamine were not effective. Another possibility is that benfotiamine reduces plaque placement by modulating the activity of glycogen synthase kinase-3 (GSK-3) [28]. Benfotiamine inhibits the three main pathways leading to hyperglycemic damage: the hexosamine pathway, the advanced glycation pathway, diacylglycerol-protein kinase C pathway, and others [29]. These pathways cause increased superoxide production [30].

In a study from Kansas City, benfotiamine was administered to non-treated alcoholics at a dose of $600 \mathrm{mg}$ or placebo over a 24week period [31]. No adverse effects were observed in 70 patients who completed the study. Consumption of alcohol has significantly decreased in both groups. The effect of benfotiamine on the reduction of drinking was shown only in women. In the follow-up study, in men, after benfotiamine administration, depression was lower if serum levels were higher [32]. Long-term administration of benfotiamine in a small study in patients with AD corrected cognitive status [21]. The study included five mild to moderately severe AD patients who received $300 \mathrm{mg}$ of benfotiamine daily for 18 months. Patients showed improvement in Mine Mental Stare Examination (MMSE) score by 3.2 points (range $0-30$, border dementia score 24) on average. Reduction of TDP stimulates neuroinflammation, enhances the effect of glycogen synthase-kinase and beta-secretase.

Our own nonpublished data in three patients confirmed the positive effect of benfotiamine in patients with even severe $\mathrm{AD}$ two male and one female who were untestable before, and after three months of benfotiamine administration in doses of 150-300 mg reached MMSE scores of 10,10 and 12.

Usual doses of benfotiamine are $150 \mathrm{mg}$ or $300 \mathrm{mg}$ once or twice daily. In some cases, Korsakoff psychosis requires long-term administration of benfotiamine (100-200 mg), with a gradual decrease in dose to maintenance dose with excellent results (unpublished case of the author). Benfotiamine can be administered to a dose of $600 \mathrm{mg}$ per day. It is often necessary to apply sustained maintenance doses after withdrawal of the symptoms in order to maintain the achieved improvement.

\section{Conclusion}

Hypovitaminosis B1 is more common than it is thought, primarily due to the discrepancy in high intake of pure calories and the low intake of vitamins characteristic of contemporary diets. The most frequent deficiencies of thiamine are Wernicke's encephalopathy, Korsakoff's psychosis, beriberi, Alzheimer's disease, but many more cases are those of marginal deficit of thiamine. Benfotiamine is a synthetic vitamin B1 that is liposoluble than manifold increases his penetration in brain and peripheral nerves compared to thiamine that can be converted into thiamine in tissues and then metabolites into thiamine monophosphate and thiamine diphosphate. The effects of benfotiamine are mild inhibition of cholinesterase, reduction in production of amyloid plaques and hyperphosphorylated tau. Long-term administration of benfotiamine corrects cognitive status. Usual doses of benfotiamine are $150 \mathrm{mg}$ or $300 \mathrm{mg}$ once or twice daily.

\section{Acknowledgements}

Funding. This study was supported by a grant from the Ministry of Science of Republic of Serbia (Project 175033).

\section{Conflict of Interest}

Author declare no financial interest or any conflict of interest.

\section{References}

1. Bubko I, Gruber BM, Anuszewska EL (2015) The role of thiamine in neurodegenerative diseases. In Polish. Postepy Hig Med Dosw (Online) 69: 1096-1106.

2. Lonsdale D (2018) Thiamin. Adv Food Nutr Res 83: 1-56.

3. Park WS, Lee J, Hong T, Park G, Youn S, et al. (2016) Comparative pharmacokinetic analysis of thiamine and its phosphorylated metabolites administered as multivitamin preparations. Clin Ther 38(10): 2277-2285.

4. Xie F, Cheng Z, Li S, Liu X, Guo X, et al. (2014) Pharmacokinetic study of benfotiamine and the bioavailability assessment compared to thiamine hydrochloride. J Clin Pharmacol 54(6): 688-695.

5. Lei Y, Zheng MH, Huang W, Zhang J, Lu Y (2018) Wet beriberi with multiple organ failure remarkably reversed by thiamine administration: A case report and literature review. Medicine (Baltimore) 97(9): e0010.

6. Guilland JC (2013) Vitamin B1 (thiamine). Rev Prat 63(8):1074-1075, 1077-1078.

7. Bunik VI (2013) Thiamin-dependent enzymes: new perspectives from the interface between chemistry and biology. FEBS J 280(24): 6373.

8. Szutowicz A, Bielarczyk H, Jankowska-Kulawy A, Pawelczyk T, Ronowska A (2013) Acetyl CoA, the key factor for survival or death of cholinergic neurons in course of neurodegenerative diseases. Neurochemical Research 2013;38(8): 1523-1542. 
9. Williams RR (1961) Toward the conquest of beriberi. Cambridge, Massachusets, Harvard University Press.

10. WHO (1999) Thiamine Deficiency and its Prevention and Control in Major Emergencies Geneva, World Health Organization.

11. Kattah JC (2017) The Spectrum of Vestibular and Ocular Motor Abnormalities in Thiamine Deficiency. Curr Neurol Neurosci Rep 17(5): 40 .

12. Lonsdale D (2012) Thiamin(e): the spark of life. Subcell Biochem 56 199-227.

13. Kerns JC, Arundel C, Chawla LS (2015) Thiamin deficiency in people with obesity. Adv Nutr 6(2): 147-153.

14. Latt N, Dore G (2014) Thiamine in the treatment of Wernicke encephalopathy in patients with alcohol use disorders. Intern Med J 44(9): 911-915.

15. Galvin R, Brathen G, Ivashynka A, Hillbom M, Tanasescu R, et al EFNS Guidelines for diagnosis, therapy and prevention of Wernicke encephalopathy. Eur J Neurol 17(12): 1408-1418.

16. Lallas M, Desai J (2014) Wernicke encephalopathy in children and adolescents. World J Pediatr 10(4): 293-298.

17. Lough ME (2012) Wernicke's encephalopathy: expanding the diagnostic toolbox. Neuropsychol Rev 22(2): 181-94.

18. Baumgartner MR (2013) Vitamin-responsive disorders: cobalamin, folate, biotin, vitamins B1 and E. Handb Clin Neurol 113: 1799-1810.

19. Costantini A, Pala MI, Compagnoni L, Colangeli M (2013) High-dose thiamine as initial treatment for Parkinson's disease. BMJ Case Rep pii: bcr2013009289.

20. Lu'o'ng K, Nguyen LT (2011) Role of thiamine in Alzheimer's disease. Am J Alzheimers Dis Other Demen 26(8): 588-598.

21. Pan X, Chen Z, Fei G, Pan S, Bao W, et al. (2016) Long-Term Cognitive Improvement After Benfotiamine Administration in Patients with Alzheimer's Disease. Neurosci Bull 32(6): 591-596.
22. Calingasan NY, Gandy SE, Baker H, Sheu KF, Kim KS, et al. (1995) Accumulation of amyloid precursor protein-like immunoreactivity in rat brain in response to thiamine deficiency. Brain Res 677(1): 50-60.

23. Gibson GE, Hirsch JA, Cirio RT, Jordan BD, Fonzetti P, et al. (2013) Abnormal thiamine-dependent processes in Alzheimer's Disease. Lessons from diabetes. Mol Cell Neurosci 55: 17-25.

24. Lonsdale D (2015) Thiamin and magnesium deficiencies: Keys to disease. Medical Hypotheses 84(2): 129-134.

25. Hoffman LaRoche F (1994) Vitamins (Basics), 1st edition, Seaboard Lithographers, New York.

26. Hawk PB, Oser BL, Summerson WH (1954) Practical physiological chemistry, 13th ed, McGraw-Hill, New York

27. Gjone E, Hakon Skramstad K (1955) Thiamin and cocarboxylase as inhibitors of cholinesterases. Acta Pharmacol Toxicol (Copenh) 11(1): 94-102.

28. Pan X, Gong N, Zhao J, Yu Z, Gu F, et al. (2015) Powerful beneficial effects of benfotiamine on cognitive impairment and b-amyloid deposition in amyloid precursor protein/presenilin-1 transgenic mice. Brain 2010:133 (Pt 5): 1342-1351.

29. Hammes HP, Du X, Edelstein D, Taguchi T, Matsumura T, et al. (2003) Benfotiamine blocks three major pathways of hyperglycemic damage and prevents experimental diabetic retinopathy. Nat Med 9(3): 294-299.

30. Nishikawa T, Edelstein D, Du XL, Yamagishi S, Matsumura T, et al. (2000) Normalizing mitochondrial superoxide production blocks three pathways of hyperglycaemic damage. Nature 404(6779): 787-790.

31. Manzardo AM, He J, Poje A, Penick EC, Campbell J, et al. (2013) Doubleblind, randomized placebo-controlled clinical trial of benfotiamine for severe alcohol dependence. Drug Alcohol Depend 133(2): 562-570.

32. Manzardo AM, Pendleton T, Poje A, Penick EC, Butler MG (2015) Change in psychiatric symptomatology after benfotiamine treatment in males is related to lifetime alcoholism severity. Drug Alcohol Depend 152: 257 263. 